\title{
Control of juvenile idiopathic scoliosis during pubertal growth spurt through conservative treatment: a retrospective observational study
}

\author{
Claudia Fusco ${ }^{*}$, Fabio Zaina, Stefano Negrini \\ From 7th International Conference on Conservative Management of Spinal Deformities \\ Montreal, Canada. 20-22 May 2010
}

\section{Background}

Juvenile idiopathic scoliosis (JIS) is characterized by an early deformity, first diagnosed between the ages of 4 and 10, that often leads to an extremely variable progression during the pubertal growth spurt (PGS). An objective of the study is to evaluate the effectiveness of conservative therapy in juvenile idiopathic scoliosis during PGS.

\section{Materials and methods}

Observational retrospective study. We enrolled 76 patients (65 females and 11 males) present in our database since 2003, with diagnosis of JIS. Mean age at first diagnosis was $6.37 \pm 1.4$. We considered as main indirect clinical sign of PGS the quick height growth (more than $5 \mathrm{~cm}$ in six months) while Risser test 1, menarche and sharp growth slowing down $(2 \mathrm{~cm}$ in six months) were considered signs of the end of PGS. According to individual needs patients were already in treatment with physical exercises alone or with brace and physical exercises. We also clustered Risser test 0 patients in 3 groups based on Cobb degrees (under $20^{\circ}$, from $20^{\circ}$ to $30^{\circ}$ and over $30^{\circ}$ ) and we observed radiographic modification during PGS. Outcome criteria: radiographic (Cobb degrees) and clinical (Bunnel, ATR, TRACE) parameters in PGS period; need to increase treatment load.

\section{Results}

$51.3 \%$ of patient worsened during PGS more than 6 Cobb degrees and the doctor decided for a treatment more effective: $28 \%$ of worsened patient changed from physical exercises to a brace ( $8 \%$ wear Spinecor and $20 \%$ a rigid

\footnotetext{
ISICO (Italian Scientific Spine Institute), Milan, Italy

Full list of author information is available at the end of the article
}

brace) while $72 \%$ of worsened patients changed in a more rigid brace or increased wearing hours, reaching 18 or 21 hours in PGS after a previous weaning phase. Cobb degrees mean thoracic values presented a statistically significant worsening from $22.9 \pm 10.9$ to $26.8 \pm 12.2$, and in lumbar curves we observed a change from $15.6 \pm 14.8$ to $16.9 \pm 15.2$ in the post treatment. We did not have statistically significant differences in TRACE, ATR and Bunnell degrees. We observed a progressive maintenance of results obtained at Risser 1 along following growing.

$10.5 \%$ overcame PGS with a curve over 45 Cobb degrees. In the subset of patients under $20^{\circ} \mathrm{Cobb}$ we observed a significant worsening and a not significant improvement in others subgroups.

\section{Discussion}

Our results confirm that the natural history of JIS lead to a worsening, often rapid, during PGS but an increase of treatment load in this period can face worsening, allowing a control of scoliosis that can be maintained until end of growth. We report an interesting result about subset in Cobb degrees: it seams that a precocious treatment can avoid a further bigger worsening.

\section{Conclusion}

Conservative treatment can effectively control JIS worsening during PGS modifying natural history.

Published: 10 September 2010

doi:10.1186/1748-7161-5-S1-074

Cite this article as: Fusco et al:: Control of juvenile idiopathic scoliosis

during pubertal growth spurt through conservative treatment: a

retrospective observational study. Scoliosis 2010 5(Suppl 1):O74. 